VOLUME 111

SEPTEMBER 13, 2007

NUMBER 36

http://pubs.acs.org/JPCB
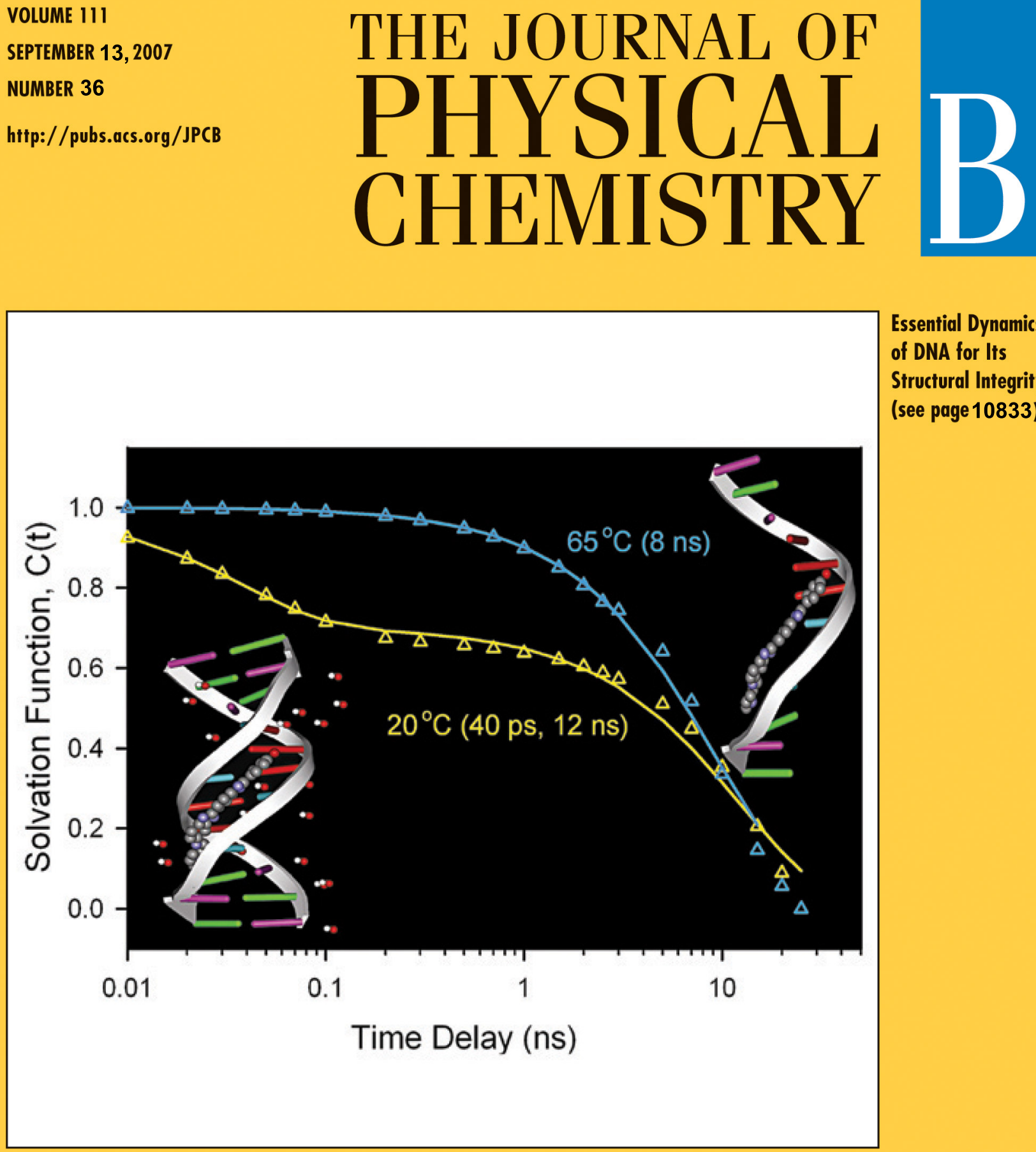

CONDENSED MATTER AND BIOPHYSICAL CHEMISTRY

Essential Dynamics of DNA for lts

Structural Integrity (see page 10833) 


\title{
Direct Observation of Essential DNA Dynamics: Melting and Reformation of the DNA Minor Groove
}

\author{
Debapriya Banerjee and Samir Kumar Pal* \\ Unit for Nano Science \& Technology, Department of Chemical, Biological \& Macromolecular Sciences, \\ S. N. Bose National Centre for Basic Sciences, Block JD, Sector III, Salt Lake, Kolkata 700 098, India
}

Received: June 18, 2007

The dynamics of bound water and ions present in the minor groove of a dodecamer DNA has been decoupled from that of the long-range twisting/bending of the DNA backbone, using the minor groove binder Hoechst 33258 as a fluorescence reporter in the picosecond-resolved time window. The bound water and ions are essential structural components of the minor groove and are destroyed with the destruction of the minor groove when the dodecamer melts at high temperatures and reforms on subsequent cooling of the melted DNA. The melting and rehybridization of the DNA has been monitored by the changes in secondary structure using circular dichroism (CD) spectroscopy. The change in the relaxation dynamics of the DNA has been studied with picosecond resolution at different temperatures, following the temperature-dependent melting and rehybridization profile of the dodecamer, using time-resolved emission spectra (TRES). At room temperature, the relaxation dynamics of DNA is governed by a $40 \mathrm{ps}(30 \%)$ and a $12.3 \mathrm{~ns}(70 \%)$ component. The dynamics of bound water and ions present in the minor groove is characterized by the 40 ps component in the relaxation dynamics of the probe bound in the minor groove of the dodecamer DNA. Analyses of the TRES taken at different temperatures show that the contribution of this component decreases and ultimately vanishes with the destruction of the minor groove and reappears again with the reformation of the groove. The dynamical behavior of bound water molecules and ions of a genomic DNA (from salmon testes) at different temperatures is also found to be consistent with that of the dodecamer. The longer component of $\approx 10 \mathrm{~ns}$ in the DNA dynamics is found to be associated with the long-range bending/twisting of the DNA backbone and the associated counterions. The transition from bound water to free water at the DNA surface, indicative of the change in the hydration number associated with each base pair, has also been ascertained in the case of the genomic DNA at different temperatures by employing densimetric and acoustic techniques.

\section{Introduction}

The dynamics of a biological macromolecule like DNA is crucial for its function. A wide variety of functions of the DNA, like recognition of proteins and small ligands, transcription, and damage recognition are associated with the structural flexibility of the polyelectrolyte. ${ }^{1-3}$ This structural flexibility originates from the movement of individual bases, sugars, and phosphate groups along with that of the water bound at the polyelectrolyte surface. A variety of techniques have been employed to characterize the dynamics of the DNA molecule over various time and length scales. ${ }^{4-9}$ NMR techniques, used to characterize the internal motions of the DNA, ${ }^{7-9}$ indicate that the internal dynamics of the DNA includes both collective long-range bending/twisting of the helix and local dynamics of the bases and backbones. The average effective correlation lifetime of these dynamics has been estimated from NMR studies to be of a few hundreds of picoseconds. A study, ${ }^{5}$ based on the temperature-dependent Stokes shift of the fluorescent probe Acridine Orange, intercalated inside DNA, showed that a substantial portion of the DNA dynamics is diffusive and coupled with the movement of the solvent. Time-resolved spectral shift (TRSS) of the Coumarin 102 molecule, artificially inserted as one of the bases of synthesized DNA, has been used in a number of studies ${ }^{10-14}$ to investigate the internal dynamics

* Corresponding author. E-mail: skpal@bose.res.in. Fax: 91332335 3477 .

10.1021/jp074697n CCC: $\$ 37.00$ of DNA. In one of the reports, it is suggested that the dynamics of DNA is spread over six decades of time, and has been expressed as a power law. ${ }^{10}$ Separate studies have shown that the internal dynamics of the DNA is slowed down in the presence of bound counterions ${ }^{14}$ and show a 5 ps component associated with the fraying at the terminal portions of the DNA. ${ }^{11}$ The relaxation dynamics of DNA, as observed in the above-mentioned studies, is smooth and lacks discernible subcomponents, ${ }^{11}$ suggesting that the motions of the different portions of the DNA are strongly coupled. Also, the similarity in the overall power law dynamics of the environment experienced by the probe molecule, inserted, respectively, in the middle and at the terminus of the DNA, shows that the relaxation is due to the immediate environment surrounding the probe and no long-range dynamics is involved. ${ }^{11}$

It is important to observe that, in all of the above-mentioned studies, an intercalated probe has been used to study the dynamics of the DNA. The artificially intercalated probe is an ideal choice to study the dynamics in the interior of the DNA. In another report, another artificially incorporated probe, 2-Aminopurine, replacing one of the adenine bases and situated at the floor separating the major and minor grooves has also been used to report the dynamics of the DNA environment, using femtosecond resolution. ${ }^{15}$ The probe in the above study ${ }^{15}$ reports a relaxation component of $10 \mathrm{ps}$ associated with the dynamics of bound water. The value agrees well with the time constant 
of $20 \mathrm{ps}$, corresponding to the dynamics of bound water, using the minor groove binder Hoechst 33258 (H258), bound to the same DNA as reported in a separate study. ${ }^{16} \mathrm{~A}$ dye, bound to the minor groove of the DNA, is exposed to the bound waters of the minor groove. ${ }^{17}$ In addition, the dye in the minor groove is in close proximity to the base pairs, and hence can experience the internal dynamics of the DNA. The relaxation dynamics of the minor groove, measured with femtosecond resolution, as reported by the well-known minor groove binder $\mathrm{H} 258,{ }^{17}$ shows a 1 ps component, characteristic of free water and a 20 ps component characteristic of bound-type water in a 200 ps window. ${ }^{16}$ In picosecond resolution, the same dye reports a 50 ps component and an $8.5 \mathrm{~ns}$ component, associated with the slow relaxation of DNA. ${ }^{18}$ Thus, it is clear that the dynamics of the minor groove, crucial for the recognition of ligand molecules in the groove, is composed of contributions from motions of free water, bound water/ions and that of the DNA backbone. However, the contributions to the environmental relaxation coming from the bound water characterizing the groove have not been decoupled from the relaxation coming from the collective slow motions of the DNA. To monitor the changes in the dynamics of the environment, experienced by the minor groove binder after the collapse of the minor groove in a dodecamer DNA, in order to decouple the contributions of groove water and monovalent cations present in the minor groove from the motions of DNA backbone is the motive of our work.

In the present communication, time-resolved emission spectra (TRES) of the fluorescent probe $\mathrm{H} 258$ has been used to characterize a synthesized dodecamer and a genomic DNA environment at different temperatures along their melting pathway. The melting and the rehybridization pathways of the dodecamer have been monitored by the circular dichroism (CD) spectra. The melting temperatures for the synthesized dodecamer and the genomic DNA have been estimated from the derivatives of the melting function. ${ }^{19}$ The binding of the $\mathrm{H} 258$ to the doubleand single-stranded DNA has been characterized by picosecondresolved polarization gated spectroscopy in the respective medium. The results show that the probe $\mathrm{H} 258$ binds to both single-stranded and double-stranded DNA, and hence is suited to report the dynamics of both native (double-stranded) and melted (single-stranded) DNA. ${ }^{20}$ The number of water molecules binding per base pair in a genomic DNA at different temperatures has been estimated by densimetric and acoustic techniques. Our results show that the dynamics of the environment includes an important contribution from bound water molecules and cations present in the minor groove of the native DNA. The contribution decreases with increasing temperature and ultimately vanishes at high temperatures, where the minor groove is destroyed as a consequence of DNA melting and the environmental relaxation is exclusively due to slow motions of the single-stranded DNA and associated counterions.

\section{Materials and Methods}

Salmon sperm DNA and phosphate buffer are purchased from Sigma. The dodecamer DNA, having the sequence CGCAAATTTGCG, is obtained from GeneLink, purified by the reverse phase cartridge technique and checked by gel electrophoresis. The gel electrophoresis result indicated a single spot consistent with pure DNA. The fluorescent dye Hoechst 33258 is obtained from Molecular Probes. All of the solutions are prepared in $50 \mathrm{mM}$ phosphate buffer using water from the Millipore system. The probe DNA solutions are prepared by adding a requisite amount of the probe in DNA and stirring for
$1 \mathrm{~h}$. The circular dichroism (CD) studies are done using a quartz cell having a path length of $1 \mathrm{~cm}$. The base pair concentrations of DNA used in the time-resolved and CD experiments are 120 $\mu \mathrm{M}$ for genomic DNA and $70 \mu \mathrm{M}$ for the dodecamer. The densimetric and acoustic studies are performed using genomic DNA having a base pair concentration of $4.2 \mathrm{mM}$.

Temperature-dependent steady-state absorption and emission are measured with a Shimadzu UV-2450 spectrophotometer and Jobin Yvon Fluoromax-3 fluorimeter, respectively, with a temperature controller attachment from Julabo (model F32). The CD measurements are done in a JASCO 815 spectropolarimeter with an attachment for the temperature-dependent measurements (Peltier). The melting temperatures of the DNAs are estimated as the temperature at which the derivative of the melting function (the melting function is obtained as a cubic polynomial fit to the central portion of the melting curve) has a maximum. ${ }^{19}$ Fluorescence transients are measured and have been fitted by using a commercially available spectrophotometer (LifeSpecps) from Edinburgh Instrument, U.K. (excitation wavelength $375 \mathrm{~nm}, 80 \mathrm{ps}$ instrument response function (IRF)), with an attachment for temperature-dependent studies. The observed fluorescence transients are fitted by using a nonlinear leastsquares fitting procedure to a function $\left(X(t)=\int_{0}^{t} E\left(t^{\prime}\right) R(t-\right.$ $\left.\left.t^{\prime}\right) \mathrm{d} t^{\prime}\right)$ comprised of convolution of the IRF $(E(t))$ with a sum of exponentials $\left(R(t)=A+\sum_{i=1}^{N} B_{i} \mathrm{e}^{-t / \tau_{i}}\right)$ with pre-exponential factors $\left(B_{i}\right)$, characteristic lifetimes $\left(\tau_{i}\right)$, and a background $(A)$. Relative concentration in a multiexponential decay is finally expressed as $c_{n}=B_{n} / \sum_{i=1}^{N} B_{i} \times 100$. The quality of the curve fitting is evaluated by reduced chi-square and residual data.

To construct time-resolved emission spectra (TRES), we follow the technique described in refs 21 and 22. As described above, the emission intensity decays are analyzed in terms of the multiexponential model:

$$
I(\lambda, t)=\sum_{i=1}^{N} \alpha_{i}(\lambda) \exp \left[-t / \tau_{i}(\lambda)\right]
$$

where $\alpha_{i}(\lambda)$ are the pre-exponential factors, with $\sum \alpha_{i}(\lambda)=1.0$. In this analysis, we compute a new set of intensity decays, which are normalized so that the time-integrated intensity at each wavelength is equal to the steady-state intensity at that wavelength. Considering $F(\lambda)$ to be the steady-state emission spectrum, we calculate a set of $H(\lambda)$ values using

$$
H(\lambda)=\frac{F(\lambda)}{\int_{0}^{\infty} I(\lambda, t) \mathrm{d} t}
$$

which for multiexponential analysis becomes

$$
H(\lambda)=\frac{F(\lambda)}{\sum_{i} \alpha_{i}(\lambda) \tau_{i}(\lambda)}
$$

Then, the appropriately normalized intensity decay functions are given by

$$
I^{\prime}(\lambda, t)=H(\lambda) I(\lambda, t)=\sum_{i=1}^{N} \alpha_{i}^{\prime}(\lambda) \exp \left[-t / \tau_{i}(\lambda)\right]
$$

where $\alpha^{\prime}{ }_{i}(\lambda)=H(\lambda) \alpha_{i}(\lambda)$. The values of $I^{\prime}(\lambda, t)$ are used to calculate the intensity at any wavelength and time, and thus the TRES. The values of the emission maxima and spectral width are determined by nonlinear least-squares fitting of the 
spectral shape of the TRES. The spectral shape is assumed to follow a log-normal line shape:

$$
I(\bar{v})=I_{0} \exp \left\{-\left[\ln 2\left(\frac{\ln (\alpha+1)}{b}\right)^{2}\right]\right\}
$$

with $\alpha=\left[2 b\left(\bar{v}-\overline{v_{\max }}\right)\right] / b>-1$, where $I_{0}$ is amplitude, $\bar{v}_{\max }$ is the wavenumber of the emission maximum, and spectral width is given by

$$
\Gamma=\Delta\left[\frac{\sinh (b)}{b}\right]
$$

The terms $b$ and $\Delta$ are asymmetry and width parameters. Equation 6 reduces to a Gaussian function for $b=0$.

The solvation correlation function $(C(t))$ is constructed following the equation

$$
C(t)=\frac{v(t)-v(\infty)}{v(0)-v(\infty)}
$$

where $v(0), v(t)$, and $v(\infty)$ stand for the wavenumber in $\mathrm{cm}^{-1}$ at the emission maxima at time zero, $t$, and infinity, respectively. For anisotropy $(r(t))$ measurements, emission polarization is adjusted to be parallel or perpendicular to that of the excitation and anisotropy is defined as

$$
r(t)=\frac{\left[I_{\text {para }}-G \times I_{\text {perp }}\right]}{\left[I_{\text {para }}+2 \times G \times I_{\text {perp }}\right]}
$$

$G$, the grating factor, is determined following the longtime tail matching technique ${ }^{23}$ to be 1.05 . The density and ultrasonic velocitimetric studies are done on a DSA 5000 instrument from Anton Paar, with the measurement accuracy and sound velocity to be $5 \times 10^{-6} \mathrm{~g} \mathrm{~cm}^{-3}$ and $0.5 \mathrm{~ms}^{-1}$, respectively. The isoentropic compressibility $(\kappa)$, which is a second pressure derivative of the Gibbs free energy, can be precisely obtained from the from the density $(\rho)$ and the velocity of sound $(u)$ in solutions by the well-known Newton Laplace equation: ${ }^{24}$

$$
\kappa=1 / \rho u^{2}
$$

In order to compare the changes in the hydration of the DNAs at different temperatures, the hydration number $\left(n_{\mathrm{h}}\right)$ has been calculated from the compressibility data based on the Passynski model:

$$
n_{\mathrm{h}}=x_{1} / x_{2}\left(1-\kappa_{2} / \kappa_{1}\right)
$$

where $x_{1}$ and $x_{2}$ represent the mole fractions and $\kappa_{1}$ and $\kappa_{2}$ represent the isoentropic compressibilities of the solvent and solute, respectively.

\section{Results and Discussion}

The B-form of DNA is a right-handed helix. The average secondary structure of DNA gives a positive peak at $277 \mathrm{~nm}$ and a negative peak around $250 \mathrm{~nm}$ in the far UV spectrum. The melting of DNA is accompanied by structural changes involving unwinding of the helix, destruction of major and minor grooves, and finally the separation of the two strands resulting in the formation of two single strands of complementary sequence. ${ }^{19}$ In our model, this is reflected by a change in the secondary structure of the DNA and the change in molar ellipticity of the DNA is utilized to construct the melting profile of the DNA. Figure 1a shows the overall secondary
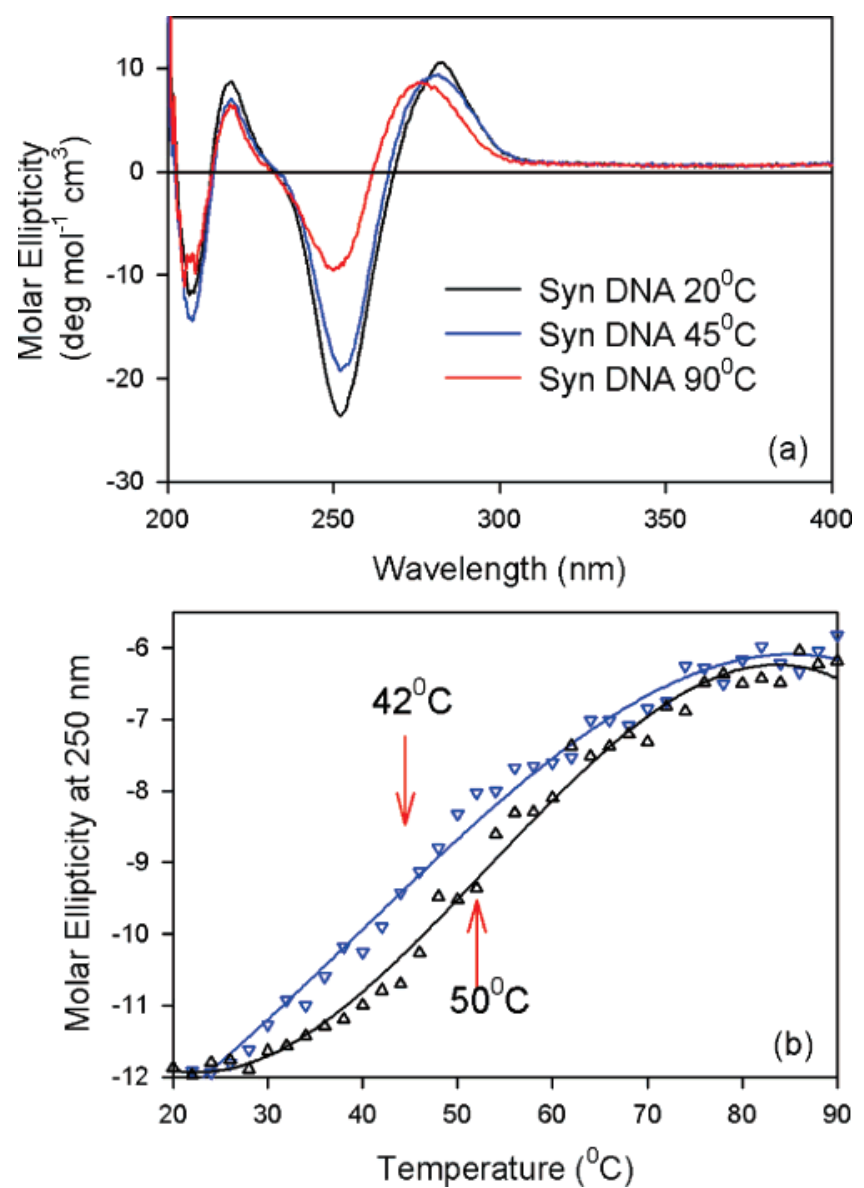

Figure 1. (a) Circular dichroism spectra of dodecamer DNA (labeled as Syn DNA in the figure) at various temperatures. (b) The melting $(\triangle)$ and rehybridization $(\nabla)$ of dodecamer DNA ([DNA] $=70 \mu \mathrm{M}$ base pair). Solid lines are the cubic polynomial fit.

structure of the dodecamer at different temperatures. It is clear that the peak at $250 \mathrm{~nm}$ is mostly affected by the temperatureinduced melting of the DNA. The change in the molar ellipticity associated with this peak has been monitored to construct the temperature-induced melting and rehybridization profiles of the dodecamer, as shown in Figure 1b. The figure shows that the dodecamer is rehybridized into the original form accompanied by a modest hysteresis effect. The melting and rehybridization temperatures have been estimated to be 50 and $42{ }^{\circ} \mathrm{C}$, respectively, in the dodecamer. In contrast, the melting and rehybridization of a genomic DNA does not follow the same pathway and there is a considerable hysteresis loss involved. This is consistent with the fact that, under our experimental conditions, the rehybridization of long strands can be erroneous due to intrastrand loop formation. ${ }^{25}$ Figure 2 a shows the change in molar ellipticity of the genomic DNA at three different temperatures, while Figure $2 \mathrm{~b}$ shows the change in molar ellipticity at $245 \mathrm{~nm}$ with the melting and rehybridization of the genomic DNA. The melting and the rehybridization temperatures of genomic DNA are found to be 83 and $58{ }^{\circ} \mathrm{C}$, respectively. It is evident from the $\mathrm{CD}$ studies that the melting of the DNA is associated with structural changes in both the dodecamer and genomic DNA. Since the structure of a biomolecule is strongly correlated with its dynamics, ${ }^{26}$ a change in the structure of the biomolecule is likely to find a reflection in the associated dynamics of the molecule. A comparison of the dynamics of the macromolecule in the native and melted states brings out the essential dynamics associated with the structure of the macromolecule. 

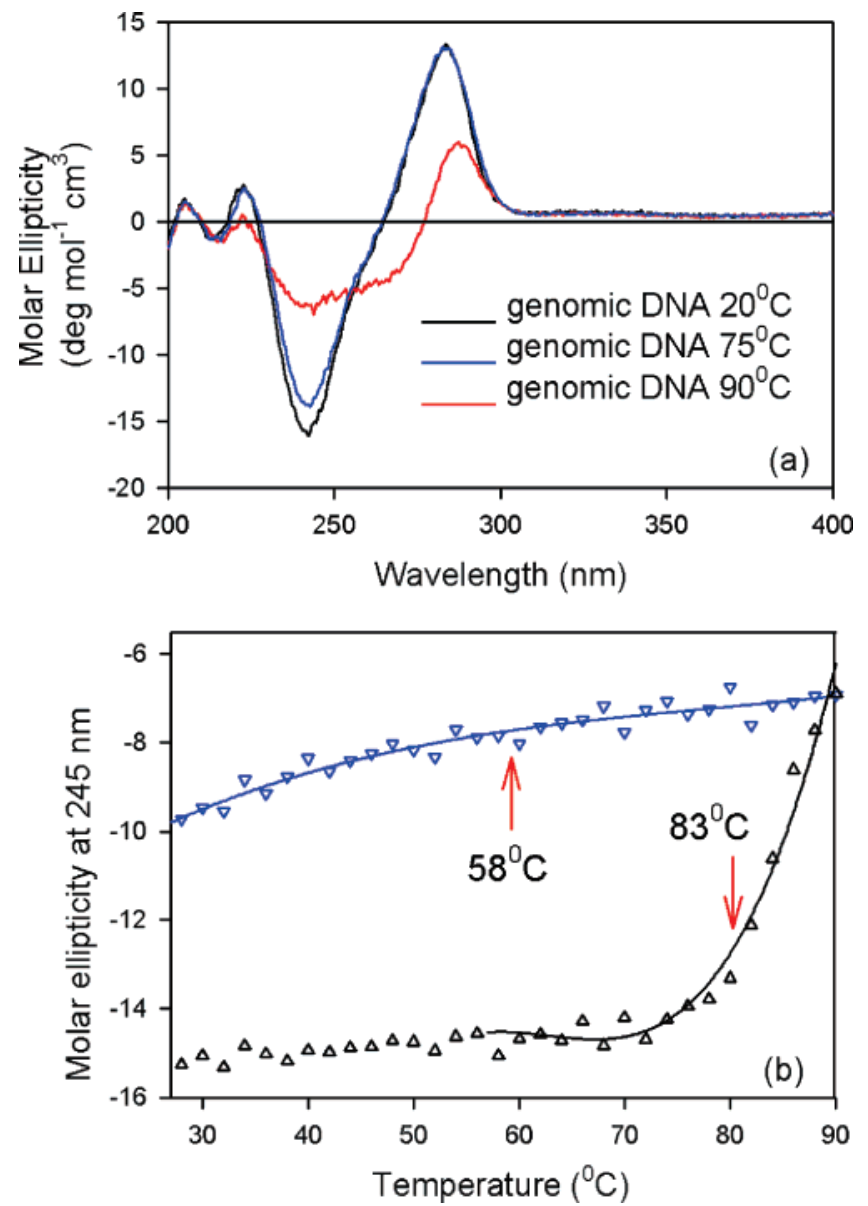

Figure 2. (a) Circular dichroism spectra of genomic DNA at various temperatures. (b) The melting $(\triangle)$ and rehybridization $(\nabla)$ of genomic DNA $([D N A]=100 \mu \mathrm{M}$ base pair). Solid lines are the cubic polynomial fit.

The solvation dynamics of the environment, constructed from the TRES measurements is an efficient technique to characterize the dynamics of a macromolecule. ${ }^{26}$ To compare the dynamics of the native and single-stranded DNA, it is essential that the fluorescence reporter remains an integral part of the DNA environment under both conditions. The anti-helmentic bisbenzimide drug $\mathrm{H} 258$ has affinity for both single-stranded and double-stranded $\mathrm{DNA}^{20}$ and hence can effectively be used as a probe to report the dynamics of DNA all along the melting profile of the DNA. The drug binds to the minor groove of the DNA as characterized by X-ray crystallographic and NMR studies. ${ }^{17,27}$ The DNA bound drug experiences a rigidity of environment, as evidenced by an increase in the time constant associated with the decay of the fluorescence anisotropy of the drug-DNA complex compared to that of the drug in bulk buffer. ${ }^{18}$ Figure 3 shows the temporal decay of fluorescence anisotropy of the drug bound to genomic and dodecamer DNA at room temperature and above the melting temperatures. The fluorescence anisotropy of the drug in bulk buffer is also shown for comparison. The fluorescence anisotropy of the drug in bulk buffer (Figure 3a) decays with a time constant of 500 ps characterizing the twisting motion of the probe in bulk buffer. ${ }^{18}$ When bound to the dodecamer at $20^{\circ} \mathrm{C}$, the absence of any 500 ps component suggests that the twisting motions of $\mathrm{H} 258$ are frozen in the dodecamer. The temporal fluorescence anisotropy of the drug at $20^{\circ} \mathrm{C}$ in the dodecamer (Figure $3 \mathrm{~b}$ ) shows a long component of $5 \mathrm{~ns}$ associated with the restricted motion of the probe in the DNA. At the higher temperature $\left(65^{\circ} \mathrm{C}\right)$, the time constant associated with the temporal decay of the
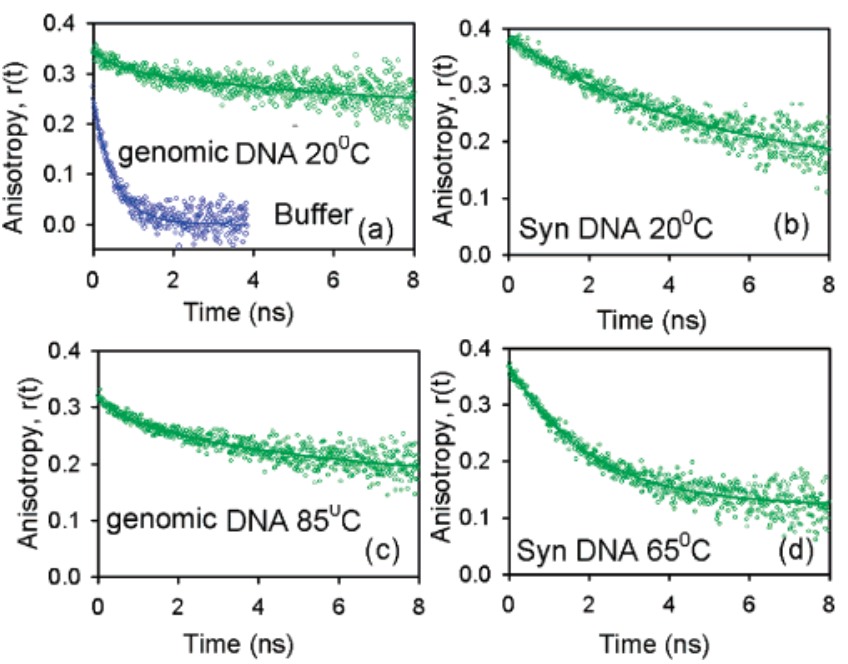

Figure 3. Fluorescence anisotropy of the $\mathrm{H} 258([\mathrm{H} 258]=1 \mu \mathrm{M}) 50$ $\mathrm{mM}$ phosphate buffer and in genomic and synthesized DNA (Syn DNA in the figures) at different temperatures. Solid lines indicate exponential fitting of the experimental data points.

fluorescence anisotropy decreases from 5 to $2 \mathrm{~ns}$, consistent with the faster rotational motions in the single-stranded DNA at high temperature. The temporal decay of the rotational anisotropy of $\mathrm{H} 258$ bound to genomic DNA at $20{ }^{\circ} \mathrm{C}$ and at $85{ }^{\circ} \mathrm{C}$ also shows similar time constants to that of the dodecamer. In addition, the temporal decay of fluorescence anisotropy in the genomic DNA shows a huge residual offset, indicating the overall motion of the DNA, which does not decay in the experimental time window. However, at all the temperatures in both the dodecamer and the genomic DNA, temporal decay of fluorescence anisotropy does not show time constants characteristic of probe in buffer. The result clearly shows that the probe is associated with the DNA even at higher temperatures and, therefore, can report the dynamics of the DNA both in the native and in the melted state.

Figure 4a shows the temporal decay of the solvation correlation function for the probe in dodecamer DNA at various temperatures. At $20^{\circ} \mathrm{C}$, the solvation correlation function decays with a time constant of $40 \mathrm{ps}(30 \%)$ and $12.3 \mathrm{~ns}(70 \%)$. At 45 ${ }^{\circ} \mathrm{C}$, the temporal decay of the solvation correlation function shows time constants of $40 \mathrm{ps}(11 \%)$ and $12.0 \mathrm{~ns}(89 \%)$. On the completion of melting, the dynamics of solvation reported by the drug shows only the nanosecond component. The retention of this component even at high temperatures when the DNA is single-stranded strongly suggests that this long component is associated with the bending and twisting motions of the DNA backbone, and strongly associated water molecules and counterions which are an integral part of the backbone. ${ }^{12,18}$ With the increase in temperature, the value of the nanosecond component decreases from 12.3 to $8.2 \mathrm{~ns}$. The decrease in the time constant associated with this nanosecond relaxation at higher temperatures could be rationalized considering the fact that with the increase in temperature the bending/twisting motion of the DNA becomes faster.

It should be noted that the short component of $40 \mathrm{ps}$ is not associated with the local motion of the H258 molecule. The rotational dynamics of the dye in bulk buffer is associated with a time constant of 500 ps. ${ }^{18}$ The time constant of $500 \mathrm{ps}$ is an order of magnitude greater than the observed time component of $40 \mathrm{ps}$. It is also to be noted that, when bound to the dodecamer, the rotational motion of the dye is completely frozen, as indicated by the absence of any subnanosecond component in the decay of the rotational anisotropy in the $\mathrm{H} 28$ bound 


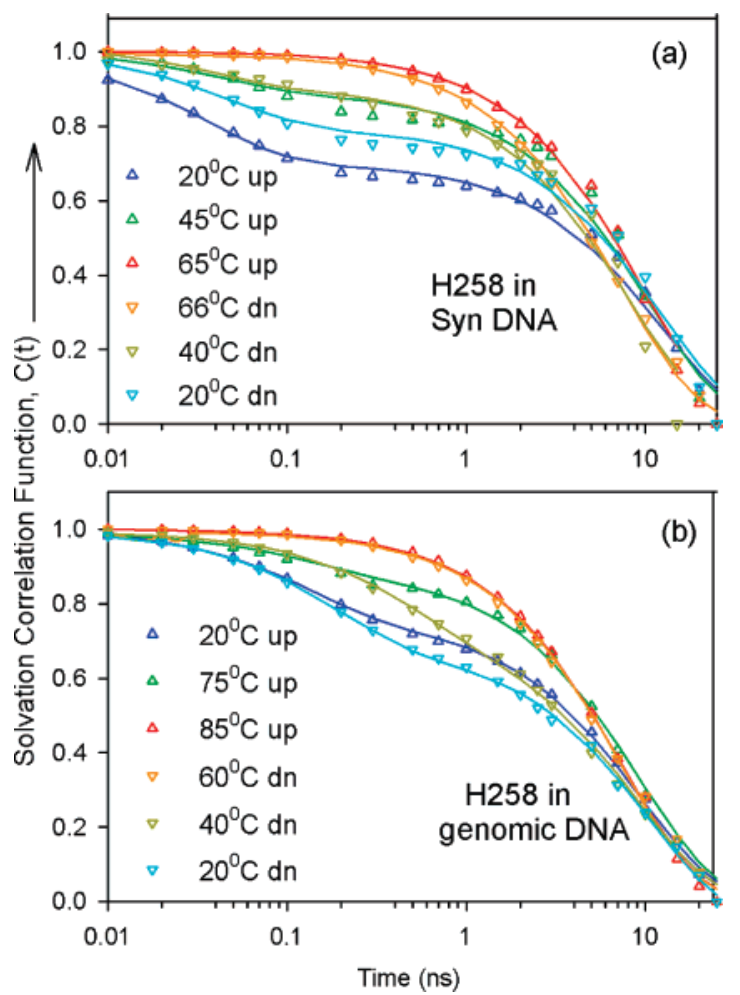

Figure 4. Temporal decay of the solvation correlation function at different temperatures for the synthesized dodecamer (labeled Syn DNA in the figure) (a) and genomic (b) DNA. The time has been plotted on a logarithmic scale. Solid lines indicate exponential fitting of the experimental data points.

dodecamer. The short component of $40 \mathrm{ps}$ is, therefore, associated with the relaxation dynamics of the DNA environment at lower temperature, which vanishes at the high temperatures, where the DNA is essentially single-stranded. This component, also, reflects the dynamics unique to the structure associated with the double-stranded DNA, namely, the base pairs and the grooves. The results from femtosecond-resolved transient absorption ${ }^{28}$ suggest an ultrafast (within $200 \mathrm{fs)} \mathrm{inertial}$ "repolarization" of nuclear degrees of freedom of an Acridine dye in the DNA pocket. In another recent study, the temperaturedependent stokes shift of the same Acridine dye intercalated in the DNA show that the DNA dynamics is essentially diffusive in nature. ${ }^{5}$ Thus, the possible contribution of the fast repolarization of base pairs to the 40 ps component in the solvation correlation function in our study can safely be ruled out. NMR studies ${ }^{29}$ have shown that the time scales associated with the opening of base pairs fall in the millisecond range; therefore, the contribution due to the opening of base pairs can also be ruled out. Thus, the observed dynamics is associated with the minor groove of the dodecamer. The femtosecond-resolved solvation studies ${ }^{16}$ of $\mathrm{H} 258$ bound to the dodecamer of the same sequence shows a 1 ps component associated with bulk water. Picosecond-resolved studies, with our instrumental resolution, on the dye in bulk water show no solvation, indicating that the experimental window associated with our studies is, indeed, blind to the dynamics of the bulk water molecules. Thus, the dynamics of free water makes no contribution to the $40 \mathrm{ps}$ component in our experimental window. The study ${ }^{16}$ also reveals a 20 ps component associated with solvation due to bound water present in the minor groove. In a recent theoretical study, ${ }^{30}$ atomistic MD simulations have been employed to determine the orientational time correlation function of water molecules bound to the minor groove in a 38 mer DNA. The study reveals that the average orientation time associated with the ordered water molecules in the minor groove is $25.8 \mathrm{ps}$. It is to be considered that the experimental window of $50 \mathrm{~ns}$ used in our studies is chosen to simultaneously report both the fast and slow dynamics of the DNA. To achieve this, the resolution of the faster components has been sacrificed. Since femtosecond timeresolved studies do not report any component in addition to the 20 ps component in a 200 ps window, it can be concluded that the 40 ps component is essentially the 20 ps component associated with the bound waters of the minor groove. Therefore, the 40 ps component, in our experimental time resolution, reflects the dynamics of the bound water in the hydration spine in the minor groove. ${ }^{31}$ It is worthwhile to mention that although the binding of the probe to the minor groove results in the expulsion of 55 water molecules from the groove, ${ }^{32}$ the spine of hydration is still present in the dodecamer-probe complex. ${ }^{33}$ A high resolution crystallographic study ${ }^{31}$ has demonstrated that monovalent cations are an intrinsic part of minor groove structure and solvation. Therefore, the possible contribution from the reorientation of these cations present in the hydration spine of the minor groove to this $40 \mathrm{ps}$ component cannot be ruled out. ${ }^{31}$

In order to investigate the nature of variation of this component, it is useful to summarize the changes associated with the melting of DNA. The melting of the DNA has been a subject of study for a long time. Various experimental and computational techniques ${ }^{34-37}$ have been employed to characterize the melting of genomic and synthesized DNAs. It is evident from these studies that the melting of a DNA duplex is associated with the unstacking and final separation of the DNA base pairs and the eventual formation of single-stranded DNA. ${ }^{19}$ The melting of DNA is associated with a positive $\Delta C_{p}$ value of $64.6 \mathrm{cal} \mathrm{deg}^{-1} \mathrm{~mol}^{-1}$ and accompanied by loss in the number of water molecules bound to the DNA. ${ }^{37}$ In a separate report, ${ }^{34}$ it has been suggested that that main contribution to the enthalpy of the process of heat denaturation of DNA duplex is the enthalpy of the disruption of the ordered water structure in the hydration shell of the double helix. The melting of DNA also results in the destruction of the minor groove and the spine of hydration associated with it. ${ }^{36}$ As the DNA melts, the bound water associated with the $40 \mathrm{ps}$ component to relaxation of DNA attains bulklike character across an energy barrier of $2.3 \mathrm{kcal}$ $\mathrm{mol}^{-1} .^{26}$ The observed energy barrier is close to the maximum value of the activation energy $\left(20 \mathrm{~kJ} \mathrm{~mol}^{-1}\left(4.7 \mathrm{kcal} \mathrm{mol}^{-1}\right)\right)$ for the diffusion of bound waters of the minor groove from the DNA surface, obtained from NMR and molecular modeling experiments. ${ }^{38}$ Thus, the initial decrease and subsequent absence of the $40 \mathrm{ps}$ component at higher temperatures further confirm that this component is associated with the dynamics of the bound water and counterions associated with the spine of hydration in the minor groove. The decrease in the spectral shift (data not shown), of $200 \mathrm{~cm}^{-1}$, associated with solvation at elevated temperatures confirms that we are losing a considerable fraction of solvation due to the conversion of bound water to bulk water. The time constants associated with the temporal decays of the solvation correlation function (Figure 4a) at decreasing temperatures following the rehybridization of the dodecamer DNA show the recovery of the $40 \mathrm{ps}$ component at the onset of structural changes associated with rehybridization. The loss in the percentage contribution of the $40 \mathrm{ps}$ component at $20^{\circ} \mathrm{C}$ in the rehybridized DNA could be due to the improper formation (not like the native DNA) of the minor groove due to a bound dye molecule. It further confirms that the structure of the minor groove is crucial for the associated dynamics. The observations show that the 40 ps component associated with the dynamics 
of bound water molecules and monovalent cations is crucial to the formation of the minor groove and is strongly correlated to the minor groove structure.

To render an interesting comparison, the same probe in genomic DNA is used to monitor the dynamics of the environment with increasing and decreasing temperature. Two widely different solvation time scales, $100 \mathrm{ps}$ and $8 \mathrm{~ns}$, again characterize (Figure $4 b$ ) the environment of the minor groove, consistent with the solvation due to the bound waters and ions present in the hydration spine and that due to the motion of the DNA backbone and ion atmosphere. The 100 ps component follows the same general trend as that followed by the $40 \mathrm{ps}$ component in the dodecamer DNA. Densimetric and acoustic measurements, used to estimate the relative change in the number of water molecules associated with a single base pair of the genomic DNA at different temperatures, reveal that there is a loss of four water molecules per base pair from 20 to $70{ }^{\circ} \mathrm{C}$, consistent with other studies. ${ }^{35}$ The 100 ps component in this case can also be said to reflect the contribution of bound water molecules, which are released at high temperatures and reformed at lower temperatures. This consistency of the trend of the picosecond component, characterizing the bound water dynamics, in both synthesized and genomic DNA gives our interpretations a more general basis. It is interesting to observe that although the average secondary structure of native and rehybridized genomic DNA shows a wide disparity (Figure 2b), it is not reflected in the dynamics reported by the dye in the rehybridized genomic DNA. The dye reports the same environment in both the native and rehybridized DNA. This interesting result can be justified considering the fact that, among the many minor grooves formed on the rehybridized genomic DNA, the drug chooses a properly formed minor groove. In the dodecamer, however, only one minor groove is formed per DNA and the formation of a minor groove is not perfect due to the bound drug.

\section{Conclusion}

In the present communication, the dynamics crucial to the minor groove and that due to the DNA chain has been decoupled. The $40 \mathrm{ps}$ component reflects the environmental relaxation characterizing the minor groove of the dodecamer DNA and is associated with the relaxation dynamics due to the bound water molecules and cations present in the hydration spine associated with the minor groove. The component is distinct from the longer $12 \mathrm{~ns}$ component associated with the twisting/ bending motions of the sugar phosphate backbone and the rigidly held water molecules and counterions, which are present even in the single-stranded DNA. The 40 ps component disappears for the dodecamer DNA with the collapse of the groove and reappears with its formation, and thus can be used to monitor the melting and reformation of the minor groove.

Acknowledgment. D.B. thanks CSIR, India, for a fellowship. We thank DST for a financial grant (SR/FTP/PS-05/2004).

\section{References and Notes}

(1) Kim, Y.; Geiger, J. H.; Hahn, S.; Sigler, P. B. Nature 1993, 365, $512-520$.

(2) Goodman, S. D.; Nash, H. A. Nature 1989, 341, 251-254.

(3) Lilley, D. M. J. Nature 1992, 357, 282-283.

(4) Hamelberg, D.; McFail-Isom, L.; Williams, L. D.; Wilson, W. D. J. Am. Chem. Soc. 2000, 122, 10513-10520.

(5) Brauns, E. B.; Murphy, C. J.; Berg, M. A. J. Am. Chem. Soc. 1998, $120,2449-2456$.
(6) Goshen, E.; Zhao, W. Z.; Carmon, G.; Rosen, S.; Granek, R.; Feingold, M. Phys. Rev. E 2005, 71, 61920(1-6).

(7) Borer, P. N.; LaPlante, S. R.; Kumar, A.; Zanatta, N.; Martin, A.; Hakkinen, A.; Levy, G. C. Biochemistry 1994, 33, 2441-2450.

(8) Eimer, W.; Williamson, J. R.; Boxer, S. G.; Pecora, R. Biochemistry 1990, 29, 799-811

(9) Alam, T. M.; Drobny, G. Biochemistry 1990, 29, 3421-3430.

(10) Andreatta, D.; Perez Lustres, J. L.; Kovalenko, S. A.; Ernsting, N. P.; Murphy, C. J.; Coleman, R. S.; Berg, M. A. J. Am. Chem. Soc. 2005 , $127,7270-7271$.

(11) Andreatta, D.; Sen, S.; Perez Lustres, J. L.; Kovalenko, S. A. Ernsting, N. P.; Murphy, C. J.; Coleman, R. S.; Berg, M. A. J. Am. Chem. Soc. 2006, 128, 6885-6892.

(12) Brauns, E. B.; Madaras, M. L.; Coleman, R. S.; Murphy, C. J.; Berg, M. A. J. Am. Chem. Soc. 1999, 121, 11644-11649.

(13) Brauns, E. B.; Madaras, M. L.; Coleman, R. S.; Murphy, C. J.; Berg, M. A. Phys. Rev. Lett. 2002, 88, 158101 (1-4).

(14) Gearheart, L. A.; Somoza, M. M.; Rivers, E. W.; Murphy, C. J.; Coleman, R. S.; Berg, M. A. J. Am. Chem. Soc. 2003, 125, 11812-11813.

(15) Pal, S. K.; Zhao, L.; Xia, T.; Zewail, A. H. Proc. Natl. Acad. Sci U.S.A. 2003, 100, 13746-13751.

(16) Pal, S. K.; Zhao, L.; Zewail, A. H. Proc. Natl. Acad. Sci. U.S.A 2003, 100, 8113-8118.

(17) Vega, M. C.; Garcia Saez, I.; Aymami, J.; Eritja, R.; VanDer Marel, G. A.; Van Boom, J. H.; Rich, A.; Coll, M. Eur. J. Biochem. 1994, 222, $721-726$.

(18) Banerjee, D.; Pal, S. K. Chem. Phys. Lett. 2006, 432, 257-262. 590.

19) Drukker, K.; Wu, G.; Schatz, G. C. J. Chem. Phys. 2001, 114, 579-

(20) Cosa, G.; Focsaneanu, K.-S.; McLean, J. R. N.; McNamee, J. P.; Scaiano, J. C. Photochem. Photobiol. 2001, 73, 585-599.

(21) Lakowicz, J. R. Principles of fluorescence spectroscopy; Kluwer Academic/Plenum: New York, 1999.

(22) Horng, M. L.; Gardecki, J. A.; Papazyan, A.; Maroncelli, M. J. Phys. Chem. 1995, 99, 17311-17337.

(23) O'Connor, D. V.; Philips, D. Time correlated single photon counting; Academic Press: London, 1984.

(24) Bockris, J. O. M.; Saluja, P. P. S. J. Phys. Chem. 1972, 76, 21402151

(25) Semsey, S.; Tolstorukov, M. Y.; Virnik, K.; Zhurkin, V. B.; Adhya, S. Genes Dev. 2004, 18, 1898-1907.

(26) Pal, S. K.; Zewail, A. H. Chem. Rev. 2004, 104, 2099-2123.

(27) Fede, A.; Labhardt, A.; Bannwarth, W.; Leupin, W. Biochemistry 1991, 30, 11377-11388.

(28) Hess, S.; Davis, W. B.; Voityuk, A. A.; Rosch, N.; Michel-Beyerle, M. E.; Ernsting, N. P.; Kovalenko, S. A.; Perez Lustres, J. L. ChemPhysChem 2002, 3, 452-455.

(29) Bhattacharya, P. K.; Cha, J.; Barton, J. K. Nucleic Acids Res. 2002, $30,4740-4750$

(30) Pal, S.; Maiti, P. K.; Bagchi, B. J. Chem. Phys. 2006, 125, $234903-$ $(1-10)$

(31) Tereshko, V.; Minasov, G.; Egli, M. J. Am. Chem. Soc. 1999, 121, 3590-3595 9794.

(32) Han, F.; Taulier, N.; Chalikian, T. V. Biochemistry 2005, 44, $9785-$

(33) Guerri, A.; Simpson, I. J.; Neidle, S. Nucleic Acids Res. 1998, 26, $2873-2878$

(34) Mrevlishvili, G. M.; Carvalho, A. P. S. M. C.; Ribeiro da Silva, M. A. V.; Mdzinarashvili, T. D.; Razmadze, G. Z.; Tarielashvili, T. Z. J. Therm. Anal. Calorim. 2001, 66, 133-144.

(35) Spink, C. H.; Chaires, J. B. Biochemistry 1999, 38, 496-508.

(36) Chen, Y. Z.; Prohofsky, E. W. Biophys. J. 1993, 64, 1385-1393.

(37) Chalikian, T. V.; Volker, J.; Plum, G. E.; Breslauer, K. J. Proc Natl. Acad. Sci. U.S.A. 1999, 96, 7853-7858.

(38) Lane, A. N.; Jenkins, T. C.; Frenkiel, T. A. Biochim. Biophys. Acta 1997, 1350, 205-220. 